\title{
The incidence of tuberculosis transmission among family members and outside households
}

Financial resources: The statutory activity of National Tuberculosis and Lung Diseases Research Institute, Task No 1.5.

\begin{abstract}
Introduction: The risk of Mycobacterium tuberculosis complex (MTBC) infection is correlated with the concentration of infectious particles and exposure time. In closed populations, healthy people staying in very frequent, close and prolonged contact with a smear-positive person, become infected and represent another link in the chain of transmission of the disease. Therefore, in the fight against tuberculosis, an important element is quick identification of the patient and potentially infected people from his environment. In epidemiological investigation of tuberculosis (TB), family members are brought under special control as they are particularly exposed to transmission of infectious diseases.

Material and methods: The study included 150 patients with bacteriologically confirmed tuberculosis who were members of 59 families. In the years 2003-2013 this population represented all TB cases detected in Poland in a family environment.Three PCR-based genotyping methods: spoligotyping, IS6110-Mtb1-Mtb2 PCR and MIRU-VNTR typing were used.

Results: Of 150 patients, 138 could be assigned to intra-household transmission on the basis of identical DNA fingerprints upon a combined typing approach. For 12 patients in 6 households, the $M$. tuberculosis isolates were clearly distinct in individual analysis - IS6110-Mtb1-Mtb2 PCR, spoligotyping or MIRU-VNTR typing or in three genotyping methods, suggesting that these patients were infected by the sources in the community.

Conclusions: The analysis confirmed the transmission of tuberculosis among members of 53 families. In the remaining 6 families the source of infection were people outside the households. In all families with young children, strains isolated from them have identical DNA patterns as strains obtained from their adult caregivers. To confirm the transmission of TB in the study population of patients, epidemiological analysis required the addition of a genotyping methods characterised by high discriminatory power.
\end{abstract}

Key words: Mycobacterium tuberculosis complex, family, transmission of tuberculosis, genotyping

Pneumonol Alergol Pol 2016; 84: 271-277

\section{Introduction}

Programmes to combat tuberculosis to much extent relies on correct diagnostic microbiology allowing to identify the patient, introduce therapy based on antibiogram and facilitate control over the spread of disease. Control and prevention of transmission of infectious diseases consist of many medical and administrative activities. Currently, the key role in determining the source of infection and tracing the chain of transmission is played by the microbiological laboratory, which, owing to genetic testing, provides irrefutable evidence for molecular affinity of bacterial strains. The employment of techniques of molecular epidemiology is possible due to recognition of genomes, marker genes and insertion sequence-elements which are used as specific molecular probes. Molecular genetic tests provide information on the spread of disease and identify epidemic outbreaks [1].

In small populations, such tests detect transmission, track the way of transmission of a patho-

Address for correspondence: Monika Kozińska, Department of Microbiology, National Tuberculosis and Lung Diseases Research Institute, Warsaw, e-mail: m.kozinska@igichp.edu.pl DOI: 10.5603/PiAP.2016.0034

Received: 28.06.2016

Copyright (C) 2016 PTChP

ISSN 0867-7077 
gen to subsequent hosts and its termination. The analysis of the disease incidence in a small population frequently allows to establish epidemiological links between patients, supplying information about their contact with their environment, thus facilitating identification of the source of transmission. The literature includes numerous reports on evidenced transmission of tuberculosis in the populations of homeless people, patients and hospital staff, in penitentiary institutions, schools and households. In such hermetic groups, healthy people are in frequent, close and long-lasting relations with patients, they are infected and thus become a consecutive chain of transmission of disease. Therefore, to fight tuberculosis, it is crucial to rapidly identify the patient and potentially infected people from the patient's surroundings [2,3].

Genetic methods are a significant supplement to epidemiologic investigation. Due to the necessity of rapid recognition of epidemic outbreaks, simple molecular techniques allowing to obtain a result within several hours are employed. Of importance is the choice of a method with highly differentiating potentiality that enables identification of genetic differences among endemic strains not belonging to the transmission outbreak under investigation.

In epidemiological investigations of tuberculosis, special attention is paid to the groups of close relatives of patients as they are particularly exposed to transmission of disease due to prolonged contact with the patient and high concentration of infectious agents in the surroundings of the smear-positive individual [4].

The present paper is the first in Poland analysis of the problem of tuberculosis transmission in family environment. The aim of this study was to answer the question: do people living close to a smear-positive family member are infected due to direct transmission, and if not, how frequent are infections from other than family sources?

\section{Material and methods}

The study group included 150 patients with bacteriologically confirmed tuberculosis diagnosed in health care centres in Poland. Microbiological data were obtained from the laboratory staff, and information about the patients (name, age, address) from the documentation collected by clinicians. The study population represented all TB cases detected among family members between 2003 and 2013. confirmed by isolating Mycobacterium tuberculosis complex strains.
The subjects came from 59 families. In 40 families, 2 tuberculosis patients were identified, in 10 families -3 patients, in 2 families -4 patients, in 4 families -5 patients, in 2 families -6 patients and in 1 family consisting of 15 members 12 people with tuberculosis were diagnosed. The subjects from one family lived together in one house/ flat and/or maintained close and frequent contact.

Taking into account degree of consanguinity, in 31 families tuberculosis affected parents and children, in 13 families - only siblings, 11 marriages, in 2 families it was diagnosed in grandparents and grandchildren, and in 2 families - in grandparents, their children and grandchildren.

The results of smear microscopy (AFB, Acid Fast Bacilli) stained with the Ziehl-Neelsen (Z.-N.) method were evaluated.

The strains were assigned to M. tuberculosis on the basis of niacin test and DNA strip assay, GenoTypeMTBC (Hain Lifescience, Germany). Drug-sensitivity of $M$. tuberculosis isolates was determined using GenoTypeMTBDRplus/sl (Hain Lifescience, Germany) and on the L.-J. medium in accordance with the methodology used in all laboratories in Poland [5].

DNA was isolated from the culture on L.-J. medium using cetyl trimethylammonium bromide (CTAB) [6].

Genotyping of $M$. tuberculosis isolates was made in accordance with the methods described, i.e. spoligotyping, IS6110-Mtb1-Mtb2 PCR and MIRU-VNTR typing [7-9].

\section{Results}

Basing on data from the Central Tuberculosis Register (CTR) concerning the time of registration of patients, 18 families were found, in which a person who could be a possible source of infection (a source case) for other family members was identified (Table 1).

A patient registered in the CTR as the first person from the family was considered to be the source case, whereas the remaining family members had tuberculosis diagnosed after at least one year. The Table illustrates consecutive TB episodes in a family with many children, consisting of 15 members. The first registered person was the father who was treated since he was 16 (1961), and then in 1970, 1984, 2003 and 2007. In 1980, his children started to suffer from TB: in 1980 two sons, then in 2001 another son, in 2003 two other sons, in 2004 two daughters and three sons, and in 2007 two daughters and one son, and in 2010 - another son. During few 
Table 1. Diagram illustrating episodes of TB in a selected large family, according to the order of registration in the Central Tuberculosis Register

\begin{tabular}{ccccccccccccc}
\hline Year & Father & Son1 & Son2 & Son3 & Son4 & Son5 & Son6 & Daughter1 & Daughter2 & Son7 & Daughter3 & Son8 \\
\hline 1961 & $\bullet$ & & & & & & & & & & & \\
1970 & $\bullet$ & & & & & & & & & & \\
1980 & & & $\bullet$ & & & $\bullet$ & & & & & & \\
1984 & $\bullet$ & & & & & & & & & & \\
2001 & & $\bullet$ & & & & & & & & & & \\
2003 & $\bullet$ & & & $\bullet$ & $\bullet$ & & & & & & \\
2004 & & $\bullet$ & & & & $\bullet$ & $\bullet$ & $\bullet$ & $\bullet$ & & & \\
2005 & & & & & & & & & & $\bullet$ & & \\
2007 & $\bullet$ & & & & & & & & $\bullet$ & & $\bullet$ & \\
2010 & & & & & & $\bullet$ & & & & & & \\
\hline
\end{tabular}

First registered person

- Next TB episodes

Table 2. AFB smear status of members of 4 families with children aged 1-12 with tuberculosis

\begin{tabular}{lcccc}
\hline Family & Family member & Age & Clinical specimen & AFB \\
\hline 26 & Mother & 36 & sputum & + \\
& Son & 12 & bronchial washings & - \\
32 & Mother & sputum & sputum & + \\
& Father & 28 & sputum & - \\
& Son & 29 & sputum & - \\
36 & Mother & 12 & gastric washings & + \\
& Daughter & 29 & sputum & sputum \\
49 & Grandfather & 1 & sputum & + \\
& Son & 63 & gastric washings & - \\
\end{tabular}

decades, only the mother and two daughters did not become ill.

In 41 families it was impossible to clearly indicate the source of infection of the remaining family members. The time between registration of consecutive patients was too short and oscillated between several days and one year.

\section{AFB smear status}

Out of 150 specimens, 92 (61\%) were positive, including 21 (14\%) AFB +++, 19 (13\%) $\mathrm{AFB}++$ and 52 (35\%) AFB +. In 58 (39\%) smears, mycobacteria were not found (AFB-). In the population of patients with positive AFB, there were 4 families with children with TB aged 1-12 years (Table 2).

\section{Drug-resistance of strains}

One hundred and forty (93\%) strains sensitive to antituberculosis drugs were identified, $4(2.5 \%)$ strains were resistant to streptomycin (SM), $2(1.5 \%)$ - to isoniazid (INH), $2(1.5 \%)-$ to isoniazid + streptomycin, and $2(1.5 \%)-$ to isoniazid + rifampicin (RMP) + streptomycin + ethambutol $(\mathrm{EMB})+$ ofloxacin (OFL) (pre-XDR resistance).

Drug-resistant strains were isolated in 4 families - in the family 26 , from a sputum-positive mother $(\mathrm{AFB}+)$ and from a 12-year-old son, strains resistant to INH were cultured. In another family, from the father and 3 adult children, strains resistant to SM were isolated. In the family 54 , from a sputum-positive daughter $(\mathrm{AFB}+)$ and 
Table 3. Data about family members with drug-resistant tuberculosis

\begin{tabular}{|c|c|c|c|c|}
\hline Family & Family member & Age & AFB & Drug-resistant profile \\
\hline \multirow[t]{2}{*}{26} & Mother & 36 & + & $\mathrm{INH}^{\mathrm{R}}$ \\
\hline & Son & 12 & - & \\
\hline \multirow[t]{4}{*}{50} & Father & 58 & - & $S M^{R}$ \\
\hline & Daughter & 23 & - & \\
\hline & Son & 29 & - & \\
\hline & Son & 27 & +++ & \\
\hline \multirow[t]{2}{*}{54} & Daughter & 24 & + & pre-XDR \\
\hline & Mother & 52 & - & $\left(\mathrm{INH}^{\mathrm{R}}+\right.$ \\
\hline \multirow[t]{2}{*}{55} & Wife & 28 & - & $I N H^{R}+S M^{R}$ \\
\hline & Husband & 39 & - & \\
\hline
\end{tabular}

her mother, pre-XDR strains were isolated. From the Chechen marriage (no 55), strains resistant to $\mathrm{INH}+\mathrm{SM}$ were isolated (Table 3).

\section{Strains genotyping - tuberculosis transmission}

The analysis of molecular formulas obtained in three genotyping methods (spoligotyping + IS6110-Mtb2-Mtb2 PCR + MIRU-VNTR) allowed to identify the strains of different and identical DNA profiles. Basing on phylogenetic relationship of the examined strains, it was found that in 53 families (90\%), in which identical strains were isolated, probably transmission of tuberculosis took place or there was a common source of infection. Whereas in 6 families (10\%), in which isolated strains had individual DNA profiles, patients could be infected by different sources outside their households.

\section{Discussion}

Exposure to infection with Mycobacterium tuberculosis complex is strictly associated with the concentration of infectious agents and exposure time. A smear-positive person with the result of smear $\mathrm{AFB}+++$ is able to infect $20 \%$ of people from his or her surroundings, whereas smear-positive patients with AFB + +/AFB+ pose a threat to at least $5 \%$ of their household members [10]. Therefore, patients with positive result of bacterioscopy and bad cough are highly infectious to healthy people maintaining with them daily contact $[4,11]$. The most threatened are family members living in one home with a smear-positive person.

Although exposure to infection in the surroundings of a smear-positive person is well

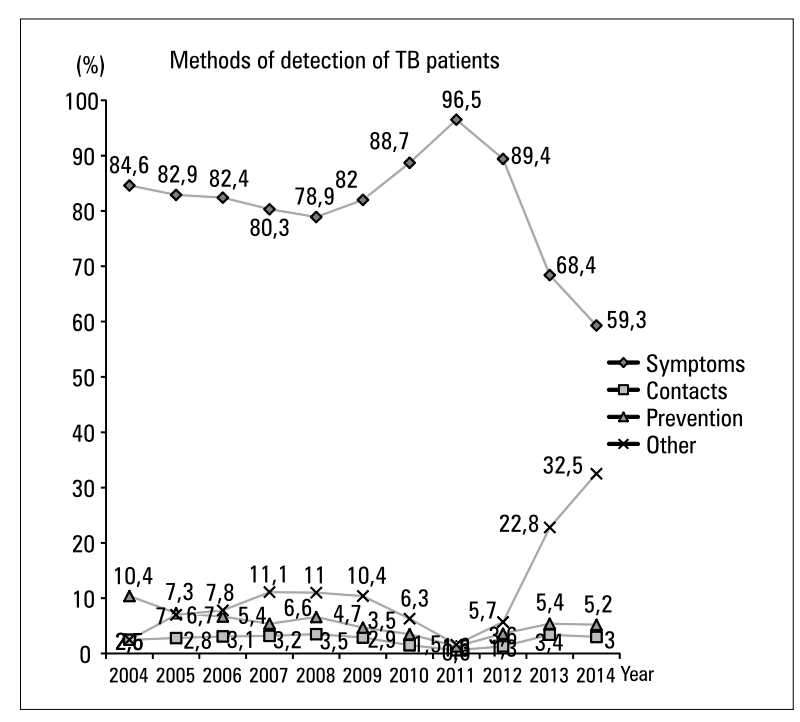

Figure 1. The effectiveness of detection methods of TB patients in Poland between 2004-2014

known, detection of people with tuberculosis in the environment of the TB patient remains poor in many countries. In Poland, some improvement in this matter has been observed recently (2012-2014), but still merely $3 \%$ of cases is detected (Fig. 1) [12].

Epidemiological investigation among people remaining in close contact with the patient are usually of passive character. Only distinctive symptoms suggesting tuberculosis, and first of all persistent bad cough force the patient to visit a doctor. Active screening programmes undoubtedly may significantly improve the outcome of detection of patients with tuberculosis [13]. However, it is believed that searching for people with tuberculosis and those infected exclusively in the closest surroundings of a smear-positive 
person is insufficient and screening programmes should reach broader categories. Besides investigations focusing on family contacts and within small, closed communities, people with tuberculosis should be sought also in the general population, particularly in the countries of high TB incidence [14].

\section{Adult to children transmission of tuberculosis}

Little children who spend at home most time and remain under surveillance of parents or custodians, are exposed to infection passed on by adults.

In children under 5 years of age, clinical symptoms of tuberculosis appear usually during the first year from infection. It may be assumed that the occurrence of tuberculosis in this group of patients indicates ongoing transmission, and a high number of cases may be the proof of some deficiencies in programmes for TB control in a given country [15].

The present paper reports also 7 families in whom, apart from adults, five children aged $1-3$ years and three children at the age of 12-14 years suffered from TB. In all cases, both in adults and living with them children, molecular formulas of the isolated strains were identical. It is worth highlighting that in the case of 4 families, high AFB smear status was found in adults who were the source cases for children.

When tuberculosis is newly diagnosed in children, the source of infection should be sought to stop transmission of disease to consecutive healthy people. However, it may be difficult to identify the source case when there are numerous sources of exposure. Detecting the real source of infection is of pivotal importance as it would allow to stop transmission and introduce proper treatment, in particular in the case of drug-resistant TB. It is of great significance in the event when TB is not microbiologically confirmed in a young patient. Then the only information about drug-resistance of the strain is supplied by the phenotype of the strain cultured from the adult who possibly was the source case [16]. In the present study, tuberculosis resistant to isoniazid was discovered in a mother of one child. The strain isolated from the child had the drug-resistance profile consistent with antibiogram of the mother's strain.

Children may be also infected by people from outside their family environment. In such situations, identification of the source of infection is usually unfeasible. The source case may come from the farther family, other children from a kindergarten, school, it may be also the person staying with the child in incidental contact - e.g. a bus driver or dental hygienist [17-19].

In the analysed material, all children under 14 years of age were infected by their parents or siblings. Some uncertainty is associated with the family from the former Soviet Union, in which an adult with TB had not been identified. Taking into consideration that this region is characterised by high incidence of TB, the children could have been infected before arrival to Poland.

\section{Transmission of drug-resistant strains}

Drug-resistant TB, particularly MDR- and XDR-TB, constitutes one of the most serious threats to health of the human population and to correctly conducted programmes for tuberculosis. It becomes extremely dangerous when identified in a family with many little children. Such families should be under specific surveillance and according to experts, control of family members and other close persons should last 2 years in the case of MDR-TB, and 4 years - in the event of XDR-TB.

In 2010, scientists from the Philippines published the results of the study conducted among families with MDR- and XDR-TB. The subjects were observed for 180 days and 30 days (the so-called epidemiological window) after introduction of treatment, and then for consecutive 4 years. It was shown that during the epidemiological window, the number of individuals with TB in the families was comparable. The differences appeared after this period and were the biggest during the first year of observation. In the families with MDR-TB, the number of new patients was diminishing with time, whereas in the families with XDR-TB, the number of persons with TB remained at a high level until the end of the four-year long observation period. The obtained results were elucidated by the fact that patients with MDR-TB respond faster to treatment and pose threat to their surroundings for shorter time [10, 20].

In the study group, in 2008, a mother and a daughter with pre-XDR-TB were detected. They are still in therapy and to date smear negativity has not been achieved. Follow-up examinations result in the MTBC culture of pre-XDR resistance and the patients remain in isolation.

\section{Child to adult transmission of tuberculosis}

Medical reports seldom document transmission of tuberculosis from children. Lack of such data is caused by the belief that children are not 
smear-positive and rarely are the source of infection to other people.

Obtaining epidemiological data concerning TB in children is problematic for several reasons: imprecise reports, difficulties in diagnosis of disease due to unspecific symptoms and low sensitivity of microbiological testing which confirms TB merely in small percentages of patients. Little children $(<5$ years) seldom expectorate sputum, and even if such material is collected, in the majority of cases bacterioscopy does not give positive results [21]. Whereas gastric washings, which are the material recommended by WHO for microbiological diagnostic techniques of TB in children, rarely give positive result in microscopic examination, and culture is obtained in maximum $50 \%$ of cases. Modern diagnostic methods are limited to large urban centres and require high qualifications of the clinical and laboratory staff. Thus, many children with TB are never diagnosed and are not included in reports.

Transmission of tuberculosis from children, although occurs rarely, is possible.

In 1999, there was a case of a 9-year-old boy who infected among others his attendant, teachers and children going with him every day by school bus [22, 23].

There are also several reports about child to adult transmission in hospitals. The source cases were over dozen-month-old children who required mechanical ventilation via endotracheal tube. The infected persons were attendants and nurses. Another report concerned a 5-year-old boy who infected 2 hospital workers [24, 25].

In the present study, 7 families with children with TB aged 1-14 years were investigated. In one of these families, a grandparent was ill and a 14-year-old granddaughter, in whom a positive result was found on bacterioscopy. It has not been confirmed who in the family was the source case, it cannot be either excluded that both patients got infection from one source outside their household.

\section{Transmission outside household}

Epidemiological investigation of TB usually concludes that transmission occurs at home. However, even two identical molecular formulas of the strains may not be sufficient proof that the source of infection is a close relative. The source sometimes may be unidentified and come from outside household.

Detection of the source is easier in the countries (e.g. Holland), where epidemiological investigations are conducted actively. It was evaluated that transmission rate between family members may amount to $31-70 \%$, whereas the source of infection comes from outside household in $18-30 \%$ of the studied contacts [26].

In the present study, 6 (10\%) families with patients infected with various tuberculosis strains were identified. This may be explained by the fact that these persons were working, socially active adults, so they could have met people with TB in their community. In such a population transmission of disease usually occurs by the so-called unconventional relations - in pubs, churches, car washes, car parks, at the hairdressers' and others, so in the circumstances, where place and time of contact are difficult to establish [27, 28].

The above reports speak in favour of molecular methods of identification of DNA profiles of M. tuberculosis. They allow to indicate possible transmission between patients not epidemiologically linked. It is estimated that genetic techniques may increase by $40 \%$ the number of detected transmissions impossible to identify with conventional methods. According to molecular standards, epidemiological investigations concerning tuberculosis employ 2 genotyping methods - spoligotyping and the MIRU-VNTR typing, which combined, assuredly identify strains belonging to a common transmission chain [29].

We applied the 3-stage molecular analysis of the strains. The results of the first step of the study proved that spoligotyping is insufficient for investigating the routes of transmission of mycobacteria - strains isolated from members of different families belong to the same spoligotypes.

Methods IS6110-Mtb1-Mtb2 PCR and MIRU-VNTR showed real relationship between strains and presented a similar discriminatory power. The same results were obtained by using them individually and together. Therefore, there is no necessary to use a three-step strategy for genotyping, but spoligotyping, as a screening method, should be supplemented with additional analysis [30],

\section{Conclusions}

Family environment is the place where transmission of tuberculosis occurs frequently. The analysis confirmed the transmission of tuberculosis among members of 53 (90\%) families. In the remaining $6(10 \%)$ families the source of infection were people outside the households. This group formed adult patients often staying away from home - at work, at meetings in pub- 
lic places. Among all households with young children, strains isolated from them presented identical DNA patterns as strains obtained from their adult caregivers. As already mentioned, the molecular relationship between strains could be the result of recent transmission, but it is necessary to examine other factors causing the clustering results, especially in situations where there is no epidemiological evidence on the link between patients. The amount of recent transmission depends heavily on the genotyping system applied, and its discriminatory power determines the proportion of clustered isolates.

\section{Conflict of interest}

The authors declare no conflict of interest.

\section{References:}

1. Desikan S, Narayanan S. Genetic markers, genotyping methods \& next generation sequencing in Mycobacterium tuberculosis. Indian J Med Res 2015; 141: 761-774.

2. Gupta V, Sugg N, Butners M, Allen-White G, Molnar A. Tuberculosis among the homeless - preventing another outbreak through community action. N Engl J Med 2015; 372: 1483-1485.

3. Dara M, Acosta CD, Melchers NV et al. Tuberculosis control in prisons: current situation and research gaps. Int J Infect Dis 2015; 32: 111-117.

4. Jones-López EC, Namugga O, Mumbowa F et al. Cough aerosols of Mycobacterium tuberculosis predict new infection: a household contact study. Am J Respir Crit Care Med 2013; 187: 1007-1015.

5. Pichula K. Wykrywanie pratków kwasoopornych metodą hodowli. W: Janowiec M. (red.). Mikrobiologia gruźlicy. PZWL, Warszawa 1977; 117-147.

6. van Embden JD, Cave MD, Crawford JT at al. Strain identification of Mycobacterium tuberculosis by DNA fingerprinting: recommendations for a standardized methodology. J Clin Microbiol 1993; 31: 406-409.

7. Kamerbeek J, Schouls L, Kolk A et al. Simultaneous detection and strain differentiation of Mycobacterium tuberculosis for diagnosis and epidemiology. J Clin Microbiol 1997; 35: 907-914.

8. Kotłowski R, Shamputa IC, El Aila NA et al. PCR-based genotyping of Mycobacterium tuberculosis with new GC-rich repeated sequences and IS6110 inverted repeats used as primers. J Clin Microbiol 2004; 42: 372-377.

9. Supply P, Mazars E, Lesjean S, Vincent V, Gicquel B, Locht C. Variable human minisatellite-like regions in the Mycobacterium tuberculosis genome. Mol Microbiol 2000; 36: 762-771.

10. Shah NS, Yuen CM, Heo M, Tolman AW, Becerra MC. Yield of contact investigations in households of patients with drug-resistant tuberculosis: systematic review and meta-analysis. Clin Infect Dis 2014; 58: 381-391.

11. Jones-López EC, Kim S, Fregona G et al. Importance of cough and M. tuberculosis strain type as risks for increased transmission within households. PLoS One 2014; 9: e100984.
12. Korzeniewska-Koseła M. (red.). Gruźlica i Choroby Układu Oddechowego w Polsce w 2014 roku. Instytut Gruźlicy i Chorób Płuc, Warszawa 2015.

13. Reichler MR, Reves R, Bur S et al. Evaluation of investigations conducted to detect and prevent transmission of tuberculosis. JAMA 2002; 287: 991-995.

14. Gilpin TP, Hammond M. Active case-finding — for the whole community or for tuberculosis contacts only? S Afr Med J 1987; 72: 260-262.

15. Schaaf HS, Michaelis IA, Richardson M et al. Adult-to-child transmission of tuberculosis: household or community contact? Int J Tuberc Lung Dis 2003; 7: 426-431.

16. Bayona J, Chavez-Pachas AM, Palacios E, Llaro K, Sapag $\mathrm{R}$, Becerra MC. Contact investigations as a means of detection and timely treatment of persons with infectious multidrug-resistant tuberculosis. Int J Tuberc Lung Dis 2003; 7: 501-509.

17. Wood R, Johnstone-Robertson S, Uys P et al. Tuberculosis transmission to young children in a South African community: modeling household and community infection risks. Clin Infect Dis 2010; 5: 401-408.

18. Merte JL, Kroll CM, Collins AS, Melnick AL. An epidemiologic investigation of occupational transmission of Mycobacterium tuberculosis infection to dental health care personnel: Infection prevention and control implications. J Am Dent Assoc 2014; 145: 464-471.

19. Yusuf HR, Braden CR, Greenberg AJ, Weltman AC, Onorato IM, Valway SE. Tuberculosis transmission among five school bus drivers and students in two New York countries. Pediatrics 1997, 100: E9.

20. Yan LP, Qin LH, Zhang Q, Sun H, Han M, Xiao HP. Transmission of extensively drug-resistant and multidrug resistant Mycobacterium tuberculosis in families identified by genotyping. Chin Med J 2013; 126: 521-555.

21. de Charnace G, Delacourt C. Diagnostic techniques in pediatric tuberculosis. Paediatr Respir Rev 2001; 2: 120-125.

22. Walls T, Shingadia D. Global epidemiology of pediatric tuberculosis. J Infect 2004; 48: 13-22.

23. Cardona M, Bek MD, Mills K, Isaacs D, Alperstein G. Transmission of tuberculosis from a seven-year-old child in a Sydney school. J Pediatric Child Health 1999; 35: 375-378.

24. Curtis AB, Ridzon R, Vogel R et al. Extensive transmission of Mycobacterium tuberculosis from a child. N Engl J Med 1999; 341: 1491-1495.

25. Rabalais G, Adams G, Stover B. PPD skin test conversion in health-care workers after exposure to Mycobacterium tuberculosis infection in infants. Lancet 1991; 338: 826.

26. Verver S, Warren RM, Munch Z et al. Proportion of tuberculosis transmission that takes place in households in a high-incidence area. Lancet 2004; 363: 212-4.

27. Kammerer JS, McNabb SJ, Becerra JE et al. Tuberculosis transmission in nontraditional settings: a decision-tree approach. Am J Prev Med 2005; 28: 201-207.

28. Veen J. Microepidemics of tuberculosis: the stone-in-the-pond principle. Tuber Lung Dis 1992; 73: 73-76.

29. Sia IG, Buckwalter SP, Doerr KA et al. Genotypic characteristics of Mycobacterium tuberculosis isolated from household contacts of tuberculosis patients in the Philippines. BMC Infect Dis 2013; 13: 571

30. Sajduda A, Dziadek J, Kotłowski R, Portaels F. Evaluation of multiple genetic markers for typing drug-resistant Mycobacterium tuberculosis strains from Poland. Diagn Microbiol Infect Dis 2006; 55: 59-64. 